\title{
Sesgos de Memoria en los Trastornos de Ansiedad
}

\section{Memory Bias in Anxiety Disorders}

\author{
Rubén Sanz Blasco, Juan José Miguel-Tobal y M. ${ }^{a}$ Isabel Casado Morales \\ Universidad Complutense de Madrid, España
}

\begin{abstract}
Resumen. En la actualidad existen un gran número de modelos teóricos que defienden la importancia de la valoración cognitiva en el inicio y mantenimiento de la respuesta de ansiedad. La investigación acerca de los procesos cognitivos que subyacen a la respuesta de ansiedad ha puesto de manifiesto de manera bastante sólida cómo los sujetos ansiosos en comparación con sujetos normales muestran una tendencia a atender de manera selectiva y a interpretar de un modo catastrofista información congruente con su estado emocional. Sin embargo, existiría un tercer sesgo para el cual los datos de las distintas investigaciones han arrojado resultados difusos en los distintos trastornos y tareas experimentales. Nos referimos al sesgo de memoria, que puede definirse como la tendencia a recordar preferentemente estimulación negativa presentada previamente en comparación con estimulación neutra. Se presenta un trabajo de revisión teórica sistemática que tiene como objetivo fundamental determinar la existencia del sesgo de memoria a lo largo de los diferentes trastornos de ansiedad y de las diferentes tareas experimentales utilizadas en la evaluación de dicho sesgo.
\end{abstract}

Palabras clave: Sesgos cognitivos, sesgos de memoria, trastornos de ansiedad.

\begin{abstract}
There are currently a large number of theoretical models that defend the importance of cognitive appraisal in the onset and maintenance of anxiety response. Research into the cognitive processes underlying anxiety response have clearly shown that, in comparison to normal subjects, anxious subjects display a tendency to selectively pay attention and catastrophically interpret information that is congruent with their emotional state. However, there is a third bias about which the data from the various investigations have yielded diffuse results in the different disorders and experimental tasks. This is the case of memory bias, which can be defined as the tendency to preferentially recall previously presented negative stimulation in comparison to neutral stimulation. A theoretical review is presented with the main goal of determining the existence of memory bias across diverse anxiety disorders and the different experimental tasks used to assess this bias.
\end{abstract}

Keywords: Cognitive bias, memory bias, anxiety disorders.

\section{Introducción}

En los últimos tiempos se está produciendo un esfuerzo y un interés creciente por tratar de determinar los procesos de valoración cognitiva que operan en el inicio y mantenimiento de la respuesta de

La correspondencia sobre este artículo debe enviarse a Juan José Miguel-Tobal a la Facultad de Psicología. Campus de Somosaguas, s/n. 28223. Pozuelo de Alarcón. Madrid. España. E-Mail: rubensanz@cop.es ansiedad. Los modelos cognitivos que se han propuesto para explicar la psicopatología de la ansiedad sugieren que los sujetos ansiosos se caracterizan por presentar sesgos en el procesamiento de la información emocional (Azais, 1995). La idea fundamental es que la respuesta de ansiedad depende, en parte, de la valoración e interpretación cognitiva que se realiza de una determinada estimulación externa o interna y no tanto de las características objetivas de la misma. 
La teoría de los cuatro factores de ansiedad, propuesta por Eysenck (1997) y Eysenck \& Derakshan (1997), postula que la ansiedad experimentada por un sujeto proviene fundamentalmente de cuatro fuentes de información diferentes; la valoración cognitiva de la situación externa, de las cogniciones, de la conducta y, por último, de la activación fisiológica. Las diferencias individuales en cuanto al procesamiento de estas cuatro fuentes de información determinarán el inicio y la intensidad de una determinada respuesta de ansiedad.

En el estudio de la ansiedad se ha encontrado evidencia acerca de la existencia de sesgos cognitivos (Mathews, Mackintosh \& Fulcher, 1997); concretamente sesgos atencionales e interpretativos. El sesgo atencional se define como la tendencia a focalizar la atención de un modo selectivo a la estimulación amenazante vs estimulación neutra, mientras que el sesgo interpretativo sería la tendencia a interpretar situaciones ambiguas como amenazantes (Eysenck \& Derakshan, 1997, p. 125). Así, se deduce que la presencia de estos sesgos cognitivos sobre una de las cuatro fuentes de información determinará la presencia de los diferentes trastornos de ansiedad. De este modo, aquellos pacientes que muestren sesgos atencionales e interpretativos en relación a su propia activación fisiológica presentarán trastorno de pánico sin agorafobia, aquellos que lo hagan sobre sus cogniciones presentarán trastorno obsesivo compulsivo, cuando los sesgos se produzcan en torno a su propia conducta se derivará una fobia social y, por último, si la información objeto de los sesgos es una determinada estimulación externa, el trastorno asociado será la fobia específica.

Existiría un tercer sesgo, objeto de estudio del presente trabajo, para el cual la literatura científica no ha mostrado evidencia consolidada acerca de su existencia y caracterización en los diferentes trastornos de ansiedad. Nos referimos al sesgo de memoria, que puede definirse como la tendencia a recordar preferentemente estimulación negativa presentada previamente en comparación con estimulación neutra. Mientras que han sido muchos los estudios destinados a la investigación de dicho sesgo en sujetos con trastornos afectivos como la depresión, son menos los estudios que han tratado de relacionar a éste con la ansiedad y, los que se han llevado a cabo, generalmente han arrojado resultados contradictorios y divergentes a lo largo de los diferentes trastornos y de las diferentes concepciones y paradigmas experimentales (tareas de memoria explícita vs implícita) que evalúan dicho sesgo (Bradley, Mogg, Millar, \& White, 1995; Calvo y García González, 1999; Mathews \& MacLeod, 1994; Tarsia, Power, \& Sanavio, 2003). Estos resultados generalmente se han interpretado en virtud del nivel de procesamiento que opera en el inicio de cada una de las manifestaciones psicopatológicas. La depresión parece estar mas relacionada con un sesgo más elaborativo y postatencional como es el sesgo de memoria, de un modo especial cuando el material procesado tiene propiedades de carácter autobiográfico con contenido de pérdida (para una revisión al respecto véase Blaney, 1986), mientras que en la ansiedad es posible que aparezcan sesgos que afecten a niveles más tempranos de procesamiento como es el sesgo atencional.

Williams, Watts, MacLeod \& Mathews (1997) sostienen que los sesgos en los procesos de activación automáticos se asocian con la ansiedad, pero no con la depresión, que se caracterizaría por un sesgo en los procesos controlados o de elaboración en la memoria. Algunos autores sostienen que la presencia de sesgos cognitivos atencionales, interpretativos y de memoria podrían ser un factor de vulnerabilidad emocional hacia los diferentes trastornos (Lundh, Czyzykow, \& Ost, 1997; Mathews \& MacLeod, 2005).

En el presente artículo se presenta un trabajo de revisión teórica sistemática que tiene como objetivo fundamental determinar la existencia de sesgos en el recuerdo en los diferentes cuadros de ansiedad y en las diferentes manifestaciones o expresiones de memoria. De encontrar discrepancias acerca de la presencia del sesgo en los diferentes trastornos de ansiedad, se tratarán de reflejar las explicaciones realizadas hasta la fecha en el análisis de dicha diferencia.

\section{Método}

La revisión se llevó a cabo a través de diferentes bases de datos, fundamentalmente ScienceDirect, 
Psycinfo y Pubmed de la National Library of Medicine (NLM). Las palabras clave utilizadas fueron "memory bias", "cognitive bias", "implicit memory", "explicit memory" y "anxiety disorders" como descriptores en el campo título y abstract encontrándose un número considerable de artículos acerca del tema de estudio. Dada la cantidad de trabajos encontrados acerca de los diferentes sesgos cognitivos se impusieron una serie de criterios de inclusión que redujeron la búsqueda a veinticinco de ellos para cumplir con el objetivo específico propuesto en el presente trabajo.

En primer lugar, los artículos debían ajustarse de un modo específico al tema de estudio, desechando aquellos que no estudiaran el sesgo de memoria de un modo aislado así como aquellos que incluyeran otros tópicos como el sesgo atencional y el interpretativo, a excepción de uno de ellos que decidió ser incluido dada la novedad de la tarea experimental utilizada. Se seleccionaron aquellos artículos tanto experimentales como de revisión que habían sido publicados en revistas psicológicas y bases de datos de reconocido prestigio. En tercer lugar, los estudios seleccionados debían haberse desarrollado siguiendo las pautas generales en cuanto a rigor metodológico se refiere, atendiendo de un modo especial al proceso de selección de la muestra, inclusión de los sujetos a los distintos grupos y diseño experimental utilizado. Como último criterio se impuso que los estudios seleccionados debían de haberse realizado con muestras clínicas o sujetos con elevado rasgo de ansiedad, eliminando de la revisión todos aquellos estudios realizados con sujetos sanos o no clínicos. La búsqueda no ha sido acotada atendiendo a un criterio temporal debido a la escasez de estudios que han tratado de determinar la presencia de los sesgos de memoria en los diferentes cuadros de ansiedad. Los artículos encontrados han sido publicados entre los años 1989 y 2010.

\section{Resultados de investigación en tareas de memoria explícita e implícita}

Los sesgos en los procesos de atención, memoria e interpretación así como ciertos pensamientos repetitivos, aunque son comunes en los diferentes desór- denes emocionales, varían de acuerdo a los diferentes tipos de trastornos (Mathews \& MacLeod, 2005).

En lo que respecta a la existencia del sesgo mnemónico en la concepción de memoria explícita, entendida ésta como la manifestación o la tendencia a recordar material presentado previamente mediante estrategias de recuperación consciente o deliberada, los hallazgos de las distintas investigaciones muestran resultados diferenciales a lo largo de los diversos trastornos y de las diferentes tareas experimentales.

En las distintas investigaciones revisadas podemos comprobar cómo en sujetos depresivos así como en aquellos con sintomatología mixta ansiedad-depresión, mediante tareas experimentales como el recuerdo libre, aparece de un modo consistente un sesgo en el recuerdo hacia la información negativa especialmente cuando el material es autobiográfico con contenido de pérdida o, en el segundo de los casos, cuando son palabras relacionadas con ansiedad (Gilboa-Schechtman, Erhard-Weiss, \& Jeczemien, 2002; Lim \& Kim, 2005; Rinck \& Becker, 2005; Tarsia et al., 2003).

En relación a los diferentes trastornos de ansiedad los hallazgos no son tan claros, apareciendo una gran cantidad de matices que conviene aclarar. De manera repetida a lo largo de los diferentes estudios, los sujetos con trastorno de pánico (TP) muestran sesgos de memoria explícita en tareas de recuerdo libre (Becker, Roth, Andrich, \& Margraf, 1999; Boyer, Bisserbe, \& Mialet, 1992; Lim \& Kim, 2005; Lundh et al., 1997; McNally, 1997), siendo escasos los estudios encontrados que muestran resultados negativos (Otto, McNally, Pollack, Chen, \& Rosenbaum, 1994). Aunque el sesgo de memoria se asocia de un modo bastante claro con el trastorno de pánico, no todos los pacientes con dicho trastorno muestran un recuerdo congruente con la emoción (Rapee, 1994).

Similares resultados, aunque en menor número de artículos y de un modo menos consistente, se ha encontrado en el trastorno de estrés postraumático (TEP) (Coles \& Heimberg, 2002; McNally, 1997; Vrana, Roodman, \& Beckham, 1995 ) y en el trastorno obsesivo compulsivo (TOC) (Coles \& Heimberg, 2002).

Por el contrario, los resultados de las distintas investigaciones arrojan generalmente resultados negativos en esta expresión de memoria para la fobia específica (McNally, 1997; Watts \& Coyle, 
1993) apareciendo en algunos estudios con paradigmas de detección de cambios en imágenes de amenaza (Reinecke, Becker, \& Rinck, 2010) y para la fobia social con tareas de recuerdo libre (Becker et al., 1999; Coles \& Heimberg, 2002; McNally, 1997; Rapee, McCallum, Melville, Ravenscroft, \& Rodney, 1994; Rinck \& Becker, 2005; Wenzel \& Holt, 2002), reconocimiento de caras presentadas previamente (Cloitre, Cancienne, Heimberg, Holt, \& Liebowitz, 1995; Perez-Lopez \& Woody, 2001) y reconocimiento de pares de palabras (Tuna, Tekcan, \& Topcuoglu, 2005). Sólo uno de los artículos objeto de revisión muestra un sesgo de memoria hacia la información amenazante en sujetos con fobia social que realizan una tarea de reconocimiento de caras. Los autores sostienen que el sesgo se da en mayor grado cuando la información se procesa profundamente (Coles \& Heimberg, 2005). En los últimos años, y en comparación con la mayoría de investigaciones que han focalizado su atención en el concepto de memoria explícita e implícita, están apareciendo resultados positivos en distintas estudios en los cuales se está considerando el papel de ciertos recuerdos con contenido autobiográfico en el desarrollo y mantenimiento de la fobia social (Morgan, 2010).

Las investigaciones que han tratado de encontrar el sesgo de memoria mediante tareas de memoria explícita en el trastorno de ansiedad generalizada han arrojado resultados negativos en tareas de recuerdo libre (Becker et al., 1999; Coles \& Heimberg, 2002; McNally, 1997; Otto et al., 1994) y reconocimiento (MacLeod \& McLaughlin, 1995), apareciendo de manera aislada en uno de los estudios analizados utilizando una tarea de recuerdo libre (Coles, Turk, \& Heimberg, 2007).

Las variaciones encontradas en cuanto a la presencia del sesgo de memoria a lo largo de las diferentes categorías diagnósticas es un importante aspecto que demanda explicación. En comparación con los pacientes con ansiedad generalizada, fobia social y fobia específica, aquellos con trastorno de estrés postraumático y trastorno de pánico pueden simplemente ser incapaces de evitar el recuerdo de información perturbadora, de manera que exhiben involuntariamente y sin esfuerzo un sesgo de memoria explícita hacia la amenaza (McNally, 1997).
De manera alternativa, se ha postulado que ciertas diferencias individuales en cuanto a determinadas variables neuropsicológicas podrían en parte determinar la presencia del sesgo. Otto et al., (1994) en un estudio con sujetos con trastorno de pánico y ansiedad generalizada asociaron el sesgo de memoria explícita con ciertas diferencias en cuanto a la lateralidad auditiva hemisférica. Aquellos pacientes con una mejor audición del oído derecho exhibían una ventaja en cuanto al recuerdo de material amenazante. Por el contrario, aquellos con una menor capacidad auditiva del mismo oído manifestaron una evitación del material relacionado con la amenaza. Los sesgos pueden estar asociados con una ventaja en la codificación de información amenazante que, en consecuencia, mejora el recuerdo. Estos resultados sugieren que determinadas variables neuropsicológicas pueden explicar, en parte, el modo en el cual los pacientes ansiosos procesan la estimulación congruente con la emoción.

A diferencia de los estudios llevados a cabo en memoria explícita, las tareas experimentales utilizadas en el estudio de los sesgos de memoria implícita en trastornos de ansiedad han sido más heterogéneas y variadas. El rendimiento en tareas de memoria implícita se mide en términos de facilitación o "priming", término utilizado para referirse a la influencia que un determinado estímulo tiene en el rendimiento o ejecución subsiguiente del sistema de procesamiento (Baddeley, 1999, p.421).

Los estudios que han tratado de estudiar el sesgo mnemónico mediante tareas de memoria implícita han arrojado resultados contradictorios (Coles \& Heimberg, 2002; McNally, 1997). Salvo en algunas ocasiones, a la luz de los artículos revisados, y al igual que ocurre en la expresión de memoria explícita, se ha encontrado el sesgo de memoria en pacientes depresivos con diferentes tareas experimentales tales como la identificación perceptiva mediante taquistoscopio (Tarsia et al., 2003) y la solución de anagramas (Rinck \& Becker, 2005).

En cuanto a los diferentes trastornos de ansiedad, en diferentes estudios experimentales se ha comprobado que los pacientes con ansiedad generalizada muestran un mayor efecto priming hacia la estimulación amenazante que los sujetos control en tareas 
de identificación perceptiva mediante taquistoscopio (MacLeod \& McLaughlin, 1995) y selección de palabras relevantes (Coles et al., 2007). Resultados opuestos a los anteriores se han obtenido en el trastorno de pánico mediante tareas de completamiento de palabras (Lundh et al., 1997).

En algunas ocasiones, el sesgo de memoria se ha estudiado en sujetos con diferentes niveles en el rasgo de ansiedad sin tener en cuenta las diferentes categorías diagnósticas. Mathews, Mogg, May \& Eysenck (1989) llevaron a cabo un estudio en el cual se trataba de determinar la existencia del sesgo de memoria en sujetos con diferentes niveles en el rasgo de ansiedad. Los resultados mostraron que en la medida explícita de memoria (recuerdo libre) no se distinguía claramente aquellos sujetos clínicamente ansiosos de aquellos que no lo eran, lo cual significaba una ausencia del sesgo. Por el contrario, en la tarea de memoria implícita (completamiento de palabras) se halló la presencia del sesgo de memoria, pero sólo para un grupo el cual había sido recientemente expuesto. Los resultados sugieren que las representaciones internas para las palabras amenazantes pueden estar activadas de un modo más persistente en estados de ansiedad aunque no necesariamente mejor elaboradas. Algunas investigaciones han concluido que los sujetos ansiosos tienden a recordar más palabras emocionales en general que aquellos que no lo son (Mogg \& Mathews, 1990).

Tabla 1. Resultados de los estudios analizados

\begin{tabular}{|c|c|c|}
\hline AUTOR Y AÑO & EXPRESIÓN DE MEMORIA Y TAREA & TRASTORNOS \\
\hline \multirow[t]{2}{*}{ Mathews et al., 1989} & M. explícita: Recuerdo libre & Ansiosos clínicos (-) \\
\hline & M. implícita: completamiento palabras & Ansiosos clínicos (+) \\
\hline Mogg \& Mathews, 1990 & M. explícita & $\begin{array}{l}\text { Ansiosos recuerdan más palabras emo- } \\
\text { cionales en general }\end{array}$ \\
\hline Boyer et al., 1992 & M. explícita: Recuerdo libre & $\mathrm{TP}(+)$ \\
\hline Watts \& Coyle, 1993 & M. explícita: Recuerdo libre & Fobia específica (arañas) (-) \\
\hline Otto et al., 1994 & M. explícita & TP y TAG $(-)$ \\
\hline \multirow[t]{2}{*}{ Rapee et al., 1994} & M. explícita: Recuerdo libre & FS (-) \\
\hline & M. explícita: Recuerdo libre & Depresivos (+) FS (-) \\
\hline Cloitre et al., 1995 & M. explícita: Recuerdo libre y reconocimiento & FS (-) \\
\hline \multirow[t]{2}{*}{ MacLeod \& McLaughlin, 1995} & M. explícita: Reconocimiento & TAG (-) \\
\hline & M. implícita: Identificación perceptiva taquistoscopio & TAG $(+)$ \\
\hline Vrana et al., 1995 & M. explícita: Recuerdo libre & $\operatorname{TEP}(+)$ \\
\hline \multirow{2}{*}{ Lundh et al., 1997} & M. explícita: Recuerdo libre & $\mathrm{TP}(+)$ \\
\hline & M. implícita: completamiento palabras & $\mathrm{TP}(-)$ \\
\hline \multirow[t]{2}{*}{ McNally, 1997 (Revisión) } & M. explícita & TP y TEP (+), Fesp, FS y TAG (-) \\
\hline & M. implícita & Resultados contradictorios \\
\hline Becker et al., 1999 & M. explícita: Recuerdo libre. & TP $(+)$, TAG y FS $(-)$ \\
\hline Perez-Lopez \& Woody, 2001 & M. explícita: Reconocimiento de caras & FS (-) \\
\hline \multirow[t]{2}{*}{ Coles \& Heimberg, 2002} & M. explícita & TP, TEP y TOC (+). FS y TAG (-) \\
\hline & M. implícita & Resultados contradictorios \\
\hline \multirow[t]{2}{*}{ Gilboa-Schechtman et al., 2002} & M. explícita: Reconocimiento de caras & Ans + depresivos $(+)$ \\
\hline & & Sólo ansiosos (+) \\
\hline Wenzel \& Holt, 2002 & M. explícita: Recuerdo libre & FS (-) \\
\hline Harrison \& Turpin, 2003 & $\begin{array}{l}\text { M. implícita: Completamiento de palabras (exactitud } \\
\text { y tiempo de reacción) }\end{array}$ & $\begin{array}{l}\text { Altos y bajos rasgo de ansiedad : sesgo } \\
\text { hacia contenido no amenazante }\end{array}$ \\
\hline \multirow[t]{4}{*}{ Tarsia et al., 2003} & M. explícita: Recuerdo libre & Depresivos $(+)$ \\
\hline & & Dep+Ans $(+)$ \\
\hline & M. implícita: Identificación palabras & Depresivos (+) \\
\hline & & Dep+Ans (-) \\
\hline Coles \& Heimberg, 2005 & M. explícita: Reconocimiento de caras & $\mathrm{FS}(+)$ \\
\hline \multirow[t]{2}{*}{ Lim \& Kim, 2005} & M. explícita: Recuerdo libre & TP, depresivos y tnos. Somatomorfos $(+)$ \\
\hline & M. implícita: Identificación perceptiva taquistoscopio & TP, depresivos y tnos. Somatomorfos (-) \\
\hline Rinck \& Becker, 2005 & M. implícita: Solución de anagramas & Depresivos (+). FS (-) \\
\hline Tuna et al., 2005 & M. explícita: Recuerdo libre y reconocimiento de pares de palabras & FS $(-)$ \\
\hline \multirow[t]{2}{*}{ Coles, Turk \& Heimberg, 2007} & M. explícita: Recuerdo libre & TAG $(+)$ \\
\hline & M. implícita: Selección de palabras relevantes & TAG $(+)$ \\
\hline Morgan, 2010 (Revisión) & Memoria autobiográfica con distintos paradigmas experimentales & $\mathrm{FS}(+)$ \\
\hline Reinecke et al.,2010 & M. explícita: Tarea de detección de cambios & Fobia específica (arañas) (+) \\
\hline
\end{tabular}


Sin embargo, estos resultados no aparecen de un modo consistente en otros trabajos incluso utilizando las mismas tareas experimentales. En algunas investigaciones se ha encontrado, contra todo pronóstico, que tanto los sujetos con niveles elevados y bajos en el rasgo de ansiedad utilizando una tarea de completamiento de palabras muestran un sesgo de memoria hacia la información no amenazante (Harrison \& Turpin, 2003).

En la tabla 1 se presenta un resumen de los resultados obtenidos en las distintas investigaciones seleccionadas en el presente artículo ordenadas en orden cronológico. Los símbolos (+) y (-) indican de manera respectiva la presencia o ausencia del sesgo de memoria. TP: Trastorno de pánico. TAG: Trastorno de ansiedad generalizada. TOC: Trastorno obsesivo-compulsivo. FS: Fobia social. TEP: Trastorno de estrés postraumático.

\section{Conclusiones y discusión}

A lo largo del presente trabajo hemos tratado de estudiar la presencia del sesgo de memoria a lo largo de los diferentes trastornos de ansiedad y de las diferentes tareas de memoria (explícita e implícita) que miden dicho sesgo.

A la luz de las investigaciones revisadas, y en consonancia con los resultados de la mayoría de las investigaciones en el campo, se observa la presencia del sesgo de memoria en el trastorno depresivo tanto en tareas de memoria explícita como implícita.

En cuanto a los trastornos de ansiedad, las conclusiones que podemos extraer son más limitadas con una mayor consistencia en unos trastornos que en otros. En cuanto a la memoria explícita, encontramos la presencia del sesgo en el trastorno de pánico, fundamentalmente con tareas de recuerdo libre. De igual manera, aparecen resultados positivos, aunque de menor peso, para el trastorno de estrés postraumático y el trastorno obsesivo-compulsivo.

En cuanto a la expresión de memoria implícita, además de existir un menor número de investigaciones que evalúen dicho sesgo, los resultados suelen ser contradictorios en los diversos trabajos, arrojando por lo general un mayor número de interrogantes que de respuestas. En algunos estudios se ha com- probado que los pacientes con ansiedad generalizada muestran un mayor efecto priming hacia la estimulación amenazante que los sujetos control en tareas de identificación perceptiva mediante taquistoscopio y selección de palabras relevantes. Mediante tareas de completamiento de palabras no se ha encontrado efectos de facilitación mnemónica en el trastorno de pánico.

A la hora de interpretar estos resultados conviene tener en cuenta una serie de aspectos que determinan el estudio del sesgo de memoria. El primer problema con el que nos encontramos a la hora de estudiar los sesgos de memoria en los trastornos de ansiedad es la alta correlación existente entre las medidas de ansiedad y depresión, correlaciones a veces más elevadas que las que se producen entre algunos cuestionarios de ansiedad, y la elevada comorbilidad entre trastornos afectivos y de ansiedad. Puesto que existe evidencia sólida acerca de la existencia de sesgos de memoria congruentes con la información emocional en trastornos depresivos, la crítica que se ha realizado a muchos estudios que han tratado de relacionar este sesgo con la ansiedad es que los resultados encontrados pueden deberse al componente de depresión presente en los trastornos de ansiedad y, no tanto, a la presencia en los mismos de sesgos en el recuerdo de naturaleza específica.

Otro de los problemas con el que nos enfrentamos en el estudio de los sesgos de memoria es que habitualmente el material utilizado en las diferentes tareas experimentales es de naturaleza verbal, generalmente palabras que son representativas de los temores de un individuo con un determinado trastorno. Sin embargo, lo más probable, es que las respuestas que acompañan a la presencia estimular de las palabras sean muy diferentes a las respuestas a las mismas amenazas en la vida real, pudiendo obtenerse resultados engañosos en cualquier prueba de memoria. Estaríamos ante un claro problema de validez ecológica que podría ser resuelto atendiendo a los avances tecnológicos como la realidad virtual que permiten recrear ambientes que la persona teme, $\mathrm{y}$ que ya están siendo utilizados en el estudio de los sesgos atencionales.

En último término, y a la luz de ciertos hallazgos experimentales, es posible que muchos de los resultados obtenidos en el estudio del sesgo en el recuer- 
do puedan ser debidos a las tareas que se llevan a cabo en el laboratorio para su medición. Como regla general, y a modo de ejemplo, se ha determinado que las características físicas de los estímulos influyen más en los efectos de facilitación que en el recuerdo consciente, en el que los rasgos semánticos son más importantes (Baddeley, 1999, p.422), al igual que se tiende a recordar más información con una tarea de recuerdo con clave que de recuerdo libre, lo cual implica la imposibilidad o inadecuación de comparar resultados que se hayan llevado a cabo utilizando tareas diferentes, ya que la presencia del sesgo podría depender de las características de la prueba y no tanto de las procesos de memoria subyacentes a cada uno de los trastornos.

Se hace necesario subsanar estas dificultades en el estudio de los sesgos de memoria para clarificar y ampliar las conclusiones y hallazgos que hasta la fecha se han encontrado y optimizar en lo posible futuras investigaciones que traten de arrojar luz a este campo de estudio.

\section{Consideraciones finales: Implicaciones en el ámbito clínico}

Pensamos que un mejor conocimiento del papel que desempeñan los procesos psicológicos en la génesis, desarrollo y/o mantenimiento de las alteraciones psicopatológicas, puede ser un elemento clave tanto en la mejora de las técnicas e instrumentos de evaluación y diagnóstico como en el desarrollo de tratamientos más eficaces.

Los trabajos experimentales realizados en las dos últimas décadas, muchos de ellos en el ámbito del laboratorio, han arrojado luz sobre los complejos mecanismos que articulan la respuesta emocional humana y parecen de gran utilidad para abordar los trastornos emocionales (fundamentalmente ansiosos y depresivos), desde una perspectiva más amplia de la que habitualmente se ha venido empleando. No olvidemos que son éstos (ansiedad y depresión) los problemas psicopatológicos que con mayor frecuencia se encuentran los psicólogos clínicos y de la salud en su práctica profesional.

A su vez, esta línea de investigación, centrada en el estudio de los sesgos cognitivos, ha permitido la elaboración de modelos y teorías de carácter integrador con una potente capacidad explicativa y predictiva, como la ya mencionada teoría de los cuatro factores de los trastornos de ansiedad de M.W.Eysenck (1997), potenciando el desarrollo de nuevos estudios dirigidos a la optimización de los procedimientos terapéuticos como ha puesto recientemente de manifiesto Eysenck (2010).

\section{Extended Summary}

In recent years, interest has increased and a great effort has been made to attempt to determine the cognitive appraisal processes that operate at the onset and maintenance of anxiety response. Studies of anxiety have found evidence of the existence of cognitive biases (Mathews, Mackintosh, \& Fulcher, 1997), specifically, attentional and interpretative biases. Attentional bias is defined as the tendency to focus attention selectively on threatening stimulation versus neutral stimulation, whereas interpretative bias is the tendency to interpret ambiguous situations as threatening (Eysenck \& Derakshan, 1997, p. 125).

There is a third bias, the object of study of the present work, for which no consolidated evidence has been found in the scientific literature of its existence and nature in the range of anxiety disorders. This is the case of memory bias, which can be defined as the tendency to preferentially recall previously presented negative stimulation in comparison to neutral stimulation.

In the present article, a systematic theoretical review is presented with the main purpose of determining the existence of recall biases in the various anxiety syndromes and manifestations or expressions of memory.

\section{Method}

The review was performed by means of a range of databases, mainly ScienceDirect, Psycinfo, and Pubmed of the National Library of Medicine (NLM). The keywords used were "memory bias," "cognitive bias," "implicit memory," "explicit memory," and "anxiety disorders" as descriptors in the 
title and abstract fields, and a considerable number of articles about the study topic were found. In view of the amount of work about the various cognitive biases, a series of inclusion criteria were enforced to reduce the search to 25 works in order to fulfill the specific purpose of the present work.

Firstly, the articles had to match the study topic specifically, discarding those that did not study memory bias in isolation, as well as those that included other topics such as attentional bias and interpretative bias, except for one article that was included in view of the novelty of the experimental task employed. Secondly, both experimental and review articles that had been published in psychological journals and databases of accredited prestige were selected. Thirdly, the studies selected had to have been developed following the general guidelines of methodological rigor, with special attention paid to the process of sample selection, the inclusion of subjects from different groups, and the experimental design employed. The last criterion was that the selected studies had to have been carried out with clinical samples or subjects with high trait anxiety, eliminating from the review all the studies conducted with healthy or nonclinical subjects. The articles found were published between the years 1989 and 2010 .

\section{Research results in explicit and implicit recall tasks}

Regarding the existence of memory bias in the conception of explicit memory, using experimental tasks such as free recall, we can confirm a consistent recall bias towards negative information in depressive subjects as well as in subjects with mixed anxiety-depression symptomatology, especially when the material is autobiographic with loss-related content or, in the latter case, when the words are related to anxiety (Gilboa-Schechtman, Erhard-Weiss, \& Jeczemien, 2002; Lim \& Kim, 2005; Rinck \& Becker, 2005; Tarsia et al., 2003).

With regard to the various anxiety disorders, the findings are not so clear, and there are many nuances that should be clarified. Repeatedly across different studies, subjects with panic disorder (PD) show memory biases of explicit recall in free recall tasks
(Becker, Roth, Andrich, \& Margraf, 1999; Boyer, Bisserbe, \& Mialet, 1992; Lim \& Kim, 2005; Lundh et al., 1997; McNally, 1997), and few studies were found that report negative results (Otto, McNally, Pollack, Chen, \& Rosenbaum, 1994).

Similar results were found in posttraumatic stress disorder (Coles \& Heimberg, 2002; McNally, 1997; Vrana, Roodman, \& Beckham, 1995) and obsessivecompulsive disorder, although there were fewer articles and the findings were less consistent (Coles \& Heimberg, 2002).

In contrast, the results of the various investigations generally yield negative results in this expression of memory for specific phobias (McNally, 1997; Watts \& Coyle, 1993); in some studies, there were paradigms of change detection of threatening images (Reinecke, Becker, \& Rinck, 2010), and for social phobia with free recall tasks (Becker et al., 1999; Coles \& Heimberg, 2002; McNally, 1997; Rapee, McCallum, Melville, Ravenscroft, \& Rodney, 1994; Rinck \& Becker, 2005; Wenzel \& Holt, 2002), recognition of previously presented faces (Cloitre, Cancienne, Heimberg, Holt, \& Liebowitz, 1995; Perez-Lopez \& Woody, 2001), and recognition of pairs of words (Tuna, Tekcan, \& Topcuoglu, 2005). Only one of the articles reviewed showed a memory bias towards threatening information in subjects with social phobia who performed face recognition tasks. The authors state that the bias occurs to a greater extent when the information is processed deeply (Coles \& Heimberg, 2005). In recent years, and in comparison with most of the investigations that have focused on the concept of explicit and implicit memory, positive results are emerging in a range of studies, in which the role of certain recollections with autobiographic content is being considered with regard to the development and maintenance of social phobia (Morgan, 2010).

The investigations that have attempted to find memory bias by means of explicit recall tasks in the generalized anxiety disorder have yielded negative results in free recall (Becker et al., 1999; Coles \& Heimberg, 2002; McNally, 1997; Otto et al., 1994) and recognition tasks (MacLeod \& McLaughlin, 1995), although bias did appear in isolation in one of the studies analyzed, using a free recall task (Coles, Turk, \& Heimberg, 2007). 
In contrast to studies of explicit memory, more heterogeneous and varied experimental tasks have been used to study implicit memory biases in anxiety disorders.

With regard to the various anxiety disorders, different experimental studies have confirmed that patients with generalized anxiety display a greater priming effect towards threatening stimulation than control subjects in perceptual identification tasks using the tachistoscope (MacLeod \& McLaughlin, 1995) and relevant word selection (Coles et al., 2007). The opposite results were obtained in panic disorder using word completion tasks (Lundh et al., 1997).

Memory bias has sometimes been studied in subjects with different levels of trait anxiety without taking into account the diverse diagnostic categories. Mathews, Mogg, May and Eysenck (1989) performed a study to determine the existence of memory bias in subjects with different levels of trait anxiety. The results showed that, in the measure of explicit memory (free recall), clinically anxious subjects could not be differentiated from subjects who were not clinically anxious, meaning there was an absence of bias. In contrast, in implicit memory tasks (word completion), the presence of memory bias was observed, but only for the group that had been recently exposed. The results suggest that internal representations of threatening words may be more persistently activated in anxiety states, although they may not necessarily be better elaborated

However, these results do not appear consistently in other works, even when using the same experimental tasks. In some investigations, it was found that, in contrast to the predictions, subjects with both high and low levels of trait anxiety display memory bias towards non-threatening information when performing a word completion task (Harrison \& Turpin, 2003).

\section{Conclusions and discussion}

In view of the works reviewed, and in consonance with the results of most of the investigations in the field, the presence of memory bias is observed in depressive disorder, both in explicit and implicit recall tasks.

With regard to anxiety disorders, the conclusions reached are more limited and more consistent in some disorders than in others. With regard to explicit recall, bias was found in panic disorder, mainly in free recall tasks. Likewise, there were positive results, although with a lower weighting, for posttraumatic stress disorder and obsessive-compulsive disorder.

With regard to the expression of implicit memory, as well as the fact that there are fewer investigations that assess this bias, the various works tend to report more contradictory results, generally yielding more queries than answers. In some studies, it was found that patients with generalized anxiety display a greater priming effect towards threatening stimulation than control subjects in perceptual identification tasks using a tachistoscope and relevant word selection. Using word completion tasks, no mnemonic facilitation effects were found in panic disorder.

When interpreting these results, some determinants of the study of memory bias should be taken into account. The first problem encountered when studying memory bias in anxiety disorders is the high correlation between the measures of anxiety and depression-correlations that are sometimes higher than those occurring in some anxiety questionnaires - and the high comorbidity of affective disorders and anxiety disorders. As there is solid evidence of the existence of memory biases that are congruent with the emotional information in depressive disorders, the criticism received by many studies that have attempted to relate this bias to anxiety is that the results obtained may be due to the depressive component present in anxiety disorders and not so much to the presence of specific recall biases in anxiety disorders.

Another problem encountered in the study of memory biases is that the range of experimental tasks normally use verbal material, generally words that represent the fears of an individual with a certain disorder. However, it is more likely that the responses that accompany the stimulus present in the words are very different from the responses to the same threats in real life, thereby producing mis- 
leading results in any memory test. This is a clear problem of ecological validity that could be resolved by using technological advances such as virtual reality, which allows one to create the feared environments and which are being used to study attentional biases.

Lastly, in view of certain experimental findings, many of the results obtained in the study of recall bias may be due to the tasks performed in the laboratory in order to measure it.

It is necessary to solve these difficulties in the study of memory biases in order to clarify and expand the conclusions and current findings and to optimize as much as possible any future research that attempts to shed light on this field of study.

\section{Final considerations: Implications in the clinical sphere}

Research focused on the study of cognitive biases has led to the elaboration of models and theories of an integrative nature with powerful explanatory and predictive capacity, such as M. W. Eysenck's (1997) four-factor theory of anxiety disorders, promoting the development of new studies aimed at the optimization of the therapeutic procedures such as those recently illustrated by Eysenck (2010).

\section{Referencias}

Azais, F. (1995). Cognitive experimental approach to anxiety disorders. Encephale, 21, 597-607.

Baddeley, A. (1999). Memoria humana: Teoría y práctica. Madrid: McGraw-Hill.

Becker, E. S., Roth, W. T., Andrich, M., \& Margraf, J. (1999). Explicit memory in anxiety disorders. Journal of Abnormal Psychology, 108, 153-163.

Blaney, P.H. (1986). Affect and memory: A review. Psychological Bulletin, 99, 229-246.

Boyer, P., Bisserbe, J. C., \& Mialet, J. P. (1992). Exploration of memory disorders in anxiety states. Encephale, 18 Spec No 2, 311-314.

Bradley, B. P., Mogg, K., Millar, N., \& White, J. (1995). Selective processing of negative information: effects of clinical anxiety, concurrent depression, and awareness. Journal of Abnormal Psychology, 104, 532-536.

Calvo, M. y García, M. D. (1999). Procesos cognitivos y ansiedad en situaciones de evaluación. Ansiedad y Estrés, 5, 229-245.

Cloitre, M., Cancienne, J., Heimberg, R. G., Holt, C. S., \& Liebowitz, M. (1995). Memory bias does not generalize across anxiety disorders. Behaviour Research and Therapy, 33(3), 305-307.

Coles, M. E., \& Heimberg, R. G. (2002). Memory biases in the anxiety disorders: current status. Clinical Psychology Review, 22, 587-627.

Coles, M. E., \& Heimberg, R. G. (2005). Recognition bias for critical faces in social phobia: a replication and extension. Behaviour Research and Therapy, 43, 109-120.

Coles, M. E., Turk, C. L., \& Heimberg, R. G. (2007). Memory bias for threat in generalized anxiety disorder: the potential importance of stimulus relevance. Cognitive Behaviour Therapy, 36, 65-73.

Eysenck, M. W. (1997). Anxiety and Cognition: A Unified Theory. London: Psychology Press.

Eysenck, M. W. (2010). La teoría de los cuatro factores de los trastornos de ansiedad: Nuevas aportaciones. Conferencia dictada en la Facultad de psicología de la UCM, Mayo 2010.

Eysenck, M. W. \& Derakshan, N. (1997). Cognitive biases for the future negative events as a function of trait anxiety and social desirability. Personality and Individual differences, 22, 597-605.

Eysenck, M. W. \& Derakshan, N. (1997). Un Marco Teórico Cognitivo Para los Trastornos de Ansiedad. Ansiedad y Estrés, 3, 121-134.

Gilboa-Schechtman, E., Erhard-Weiss, D., \& Jeczemien, P. (2002). Interpersonal deficits meet cognitive biases: memory for facial expressions in depressed and anxious men and women. Psychiatry Research, 113, 279-293.

Harrison, L. K., \& Turpin, G. (2003). Implicit memory bias and trait anxiety: a psychophysiological analysis. Biological Psychology, 62(2), 97-114.

Lim, S. L., \& Kim, J. H. (2005). Cognitive processing of emotional information in depression, panic, and somatoform disorder. Journal of Abnormal Psychology, 114, 50-61.

Lundh, L. G., Czyzykow, S., \& Ost, L. G. (1997). Explicit and implicit memory bias in panic disor- 
der with agoraphobia. Behaviour Research and Therapy, 35, 1003-1014.

MacLeod, C., \& McLaughlin, K. (1995). Implicit and explicit memory bias in anxiety: a conceptual replication. Behaviour Research and Therapy, 33, 1-14.

Mathews, A., Mackintosh, B. \& Fulcher, E. (1997). Cognitive biases in anxiety and attention to threat. Journal of Abnormal Psychology, 340-345.

Mathews, A. \& MacLeod, C. (1994). Cognitive appraches to emotion and emotional disorders. Annual Review of Psychology, 45, 25-50.

Mathews, A., \& MacLeod, C. (2005). Cognitive vulnerability to emotional disorders. Annual Review of Clinical Psychology, 1, 167-195.

Mathews, A., Mogg, K., May, J., \& Eysenck, M. (1989). Implicit and explicit memory bias in anxiety. Journal of Abnormal Psychology, 98, 236240.

McNally, R. J. (1997). Memory and anxiety disorders. Philosophical Transactions of the Royal Society B: Biological Sciences, 352, 1755-1759.

Mogg, K., \& Mathews, A. (1990). Is there a self-referent mood-congruent recall bias in anxiety? Behaviour Research and Therapy, 28, 91-92.

Morgan, J. (2010). Autobiographical memory biases in social anxiety. Clinical Psychology Review, 30, 288-297.

Otto, M. W., McNally, R. J., Pollack, M. H., Chen, E., \& Rosenbaum, J. F. (1994). Hemispheric laterality and memory bias for threat in anxiety disorders. Journal of Abnormal Psychology, 103, 828-831.

Perez-Lopez, J. R., \& Woody, S. R. (2001). Memory for facial expressions in social phobia. Behaviour Research and Therapy, 39, 967-975.

Rapee, R. M. (1994). Failure to replicate a memory bias in panic disorder. Journal of Anxiety Disorders, 8, 291-300.

Rapee, R. M., McCallum, S. L., Melville, L. F., Ravenscroft, H., \& Rodney, J. M. (1994). Memory bias in social phobia. Behaviour Research and Therapy, 32, 89-99.

Reinecke, A., Becker, E. S., \& Rinck, M. (2010). Visual working memory and threat monitoring: Spider fearfuls show disorder-specific change detection. Behaviour Research and Therapy, 48, 770-778.

Rinck, M., \& Becker, E. S. (2005). A comparison of attentional biases and memory biases in women with social phobia and major depression. Journal of Abnormal Psychology, 114, 62-74.

Tarsia, M., Power, M. J., \& Sanavio, E. (2003). Implicit and explicit memory biases in mixed anxiety-depression. Journal of Affective Disorders, 77, 213-225.

Tuna, S., Tekcan, A. I., \& Topcuoglu, V. (2005). Memory and metamemory in obsessive-compulsive disorder. Behaviour Research and Therapy, 43, 15-27.

Vrana, S.R., Roodman, A., \& Beckham, J.C. (1995). Selective processing of trauma relevant words in post-traumatic stress disorder. Journal of Anxiety Disorders, 9, 515-530.

Watts, F. N., \& Coyle, K. (1993). Phobics show poor recall of anxiety words. British Journal of Medical Psychology, 66, 373-382.

Wenzel, A., \& Holt, C. S. (2002). Memory bias against threat in social phobia. British Journal of Clinical Psychology, 41, 73-79.

Williams, J.M.G., Watts, F.N., MacLeod, C. y Mathews, A. (1997). Cognitive psychology and emotional disorders. ( $2^{\text {nd }}$ ed.). Chichester, UK: Wiley.

Manuscrito recibido: 25/01/2011

Revisión recibida: 14/04/2011

Aceptado: 05/05/2011 